\title{
43. Calcification and Angioproliferation in Epicardium of Mouse induced by Mutagenic/carcinogenic Heterocyclic Amine
}

\author{
By Shozo Takayama,*) Michiie Sakamoto,**) Yasuhiro Hosoda,**। \\ and Takashi Sugimura, M. J. A.*)
}

(Communicated May 12, 1987)

Introduction. Carcinogenic polycyclic aromatic hydrocarbons, induced atherosclerosis in the abdominal aorta when injected into chickens and mice. ${ }^{1)-4}$ )

A series of new mutagenic/carcinogenic heterocyclic amines has been isolated from the pyrolysates of amino acids and proteins, and from cooked foods." Among these heterocyclic amines, 2-amino-6-methyldipyrido[1,2- $\left.a: 3^{\prime}, 2^{\prime}-d\right]$ imidazole (Glu-P-1), 2-aminodipyrido[1,2-a: $\left.3^{\prime}, 2^{\prime}-d\right]$ imidazole (Glu-P-2), 2-amino-9Hpyrido $[2,3-b]$ indole $(\mathrm{A} \alpha \mathrm{C})$ and 2-amino-3-methyl-9H-pyrido[2,3-b] indole $(\mathrm{MeA} \alpha \mathrm{C})$ have induced hemangioendothelial cell sarcomas in the intrascapular brown adipose tissue of $\mathrm{CDF}_{1}$ mice. $^{6)}$

The present paper describes that feeding Glu-P-1 to mice during 4-month experiments, induces calcification and angioproliferation in the epicardium of the right ventricle of the heart.

Materials and methods. Synthetic Glu-P-1 was obtained from Katsura Chemical Co., Tokyo, Japan. The purity of the compound was confirmed by high performance liquid chromatography, and mass and infrared spectrophotometry. Glu-P-1 was added to a pellet diet (CE-2; CLEA Japan, Tokyo) at a concentration of $500 \mathrm{ppm}$. The presence of almost all added Glu-P-1 in the diet was confirmed by high performance liquid chromatography after extraction with methanol.

Five-week-old $\mathrm{CDF}_{1}\left[(\mathrm{BALB} / \mathrm{cAnN} \times \mathrm{DBA}(2 \mathrm{~N})] \mathrm{F}_{1}\right.$ male mice were purchased from Charles River Japan, Inc., Kanagawa, Japan. They were housed 5 to a plastic cage. Food and tap water were provided ad libitum.

Total 90 mice were given a diet containing 500 ppm Glu-P-1 from 8 weeks of age. A control group of 30 mice of the same age was given the basal diet only. Ten male mice underwent autopsy, at each of 2,3 and 4 months after experiment. Control groups of 10 male mice were examined at 4 months.

Specimens of the heart with white spots were fixed in $4 \%$ paraformaldehyde in $0.1 \mathrm{M}$ phosphate buffer ( $\mathrm{pH}$ 7.4). After fixation, cryosections were made and stained for alkaline phosphatase by the azo coupling method. The histochemical staining for calcification was performed using von Kossa's method. All specimens were fixed also in $10 \%$ neutralized formalin, embedded in paraffin, processed, and stained with hematoxylin and eosin.

Results and discussion. Two months after treatment, macroscopically white lesions were found in 2 of the 10 excised hearts. They were scattered with white spots along the surface of the right ridge. Microscopic findings

*) National Cancer Center Research Institute, Tsukiji, Chuo-ku, Tokyo 104, Japan.

**) School of Medicine, Keio University, Shinano-machi, Shinjuku-ku, Tokyo 160, Japan. 
[Vol. $63(\mathrm{~B})$,

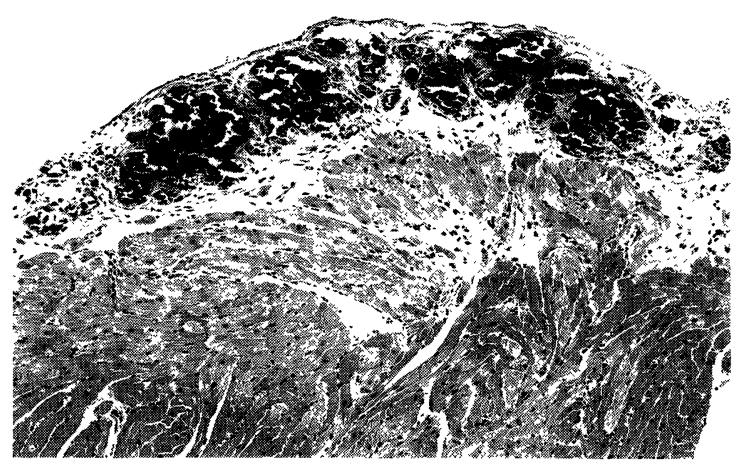

Fig. 1. Calcium foci in the visceral layer of the epicardium of the right ventricle in a mouse given the Glu-P-1 diet, examined 3 months after experiment.

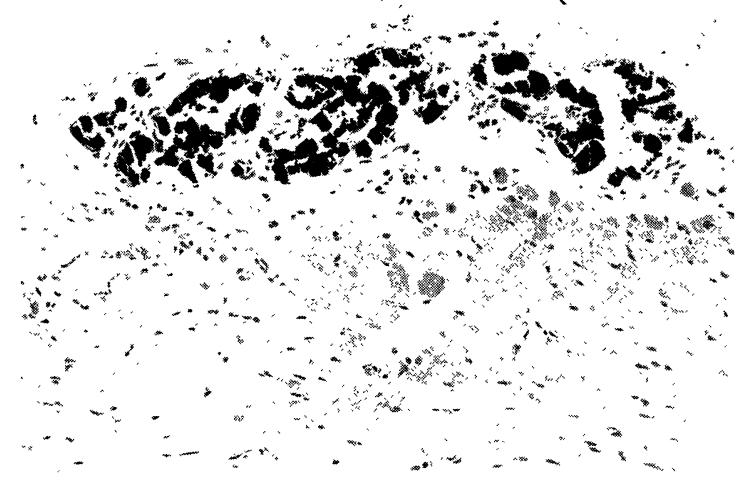

Fig. 2. von Kossa-stained section of calcium deposit foci in the epicardium of a mouse given the Glu-P-1 diet for 2 months.

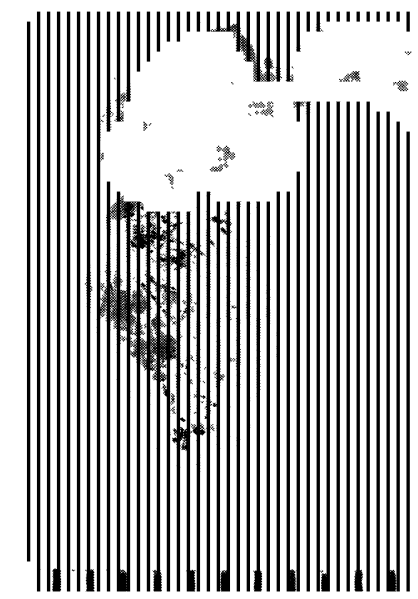

Fig. 3. A $\mathrm{CDF}_{1}$ mouse given the Glu-P-1 diet for 3 months, showing band-like calcium deposits along the coronary artery of the heart. 
showed an increased number of angio-like lumens and an associated or independent calcium foci in the visceral layer of the epicardium of the right ventricle (Fig. 1). The von Kossa-stained sections of these lesions showed some case-tocase variation. Most of the calcification occurred intraluminally or periluminally (Fig. 2).

Some of the lumens exhibited features of lymphatic capillaries and others of blood vessel capillaries. Alkaline phosphatase staining showed a strong reaction in endothelial capillary cells in the muscle layer and coronary artery. In contrast, most of the newly formed and some dilated angio-like lumen showed no positive reaction.

Seven out of 10 and 6 out of 10 excised hearts showed the same lesion macroscopically as the above, 3 and 4 months after experiment, respectively. The degree of the lesions was slightly increased by period of experiments and proceeded ones showed the fusion of the white spots and a band-like formation (Fig. 3). In fact, even in such cases, the lesions were consistent, appearing to extend along the right coronary artery. In the control group, 3 out of 10 mice showed scattered milliary sized white spots in the right ventricle of the heart macroscopically at 4 months.

It is an interesting finding that moderate atrophy of the interscapular brown adipose tissue was found at 3 and 4 months. Microscopically, only a few calcifying foci were exhibited and no angiomatous change was observed.

The pre-existence of pericarditis is unlikely from the histology, and the possible role of Glu-P-1 treatment in damaging the vessels can be proposed which induces the angioproliferation in epicardium.

The experiment is now in progress, any further studies needed on the relation calcification, atherosclerosis and hemangioendothelial sarcoma in $\mathrm{CDF}_{1}$ mice by Glu-P-1.

Acknowledgement. This work was supported by a Grant-in-Aid for Cancer Research from the Ministry of Health and Welfare, Japan.

\section{References}

1) Albert, R. et al.: Cancer Res., 37, 2232-2235 (1977).

2) Penn, A. et al.: ibid., 41, 588-592 (1981).

3) Majesky, M. W. et al.: Proc. Natl. Acad. Sci., 82, 3450-3454 (1985).

4) White, J. et al.: JNCI, 3, 453-454 (1943).

5) Sugimura, T.: Environ. Health Perspect., 67, 5-10 (1986).

6) Ohgaki, H. et al.: Carcinogenesis, 5, 815-819 (1984). 